\title{
Análisis de la Memoria Cotidiana en alumnos del Programa Universitario para Mayores en Almería
}

\author{
José J. Gázquez, Ma del Carmen Pérez-Fuentes y José J. Carrión \\ Universidad de Almería (España)
}

\begin{abstract}
Las Universidades de Mayores son uno de los programas actuales de envejecimiento activo con mayor auge en España. La participación en estos tiene una repercusión no sólo en la mejora de la integración y actividad social, sino que los aprendizajes en estas edades reportan beneficios en la salud física y mental. Así, el objetivo de nuestra investigación es analizar estos beneficios respecto a la memoria cotidiana (espacial, lingüística, secuencial y de cosas) de la asistencia a la Universidad de Mayores. Para ello la muestra está compuesta de dos grupos uno perteneciente a la Universidad de Mayores, que llevan en esta más de cinco años $(\mathrm{N}=264)$, y otro de mayores de 50 años que no pertenecen a la Universidad de Mayores $(\mathrm{N}=453)$. En una comparativa de ambos grupos, los resultados nos muestran, que los alumnos de la Universidad de Mayores obtienen puntuaciones significativamente menores en la puntuación global de memoria cotidiana, en la memoria lingüística, secuencial y de cosas.
\end{abstract}

Palabras clave: Universidad de mayores, envejecimiento activo, mayores, memoria cotidiana.

Analysis of Daily Memory in students of the University Program for Older People in Almería. Universities for older people are currently one of the most popular programs for active ageing Spain. Older people's participation in such programs has repercussions, not only in the improvement of their integration and social activity, but also in that learning at these ages produces benefits in physical and mental health. Thus, the goal of our investigation is to analyze these benefits in daily memory (spatial, linguistic, sequential, and of things) from attending the University for Older People. For this purpose, the sample is made up of two groups, one from the University for Older People, who have been attending it for more than five years $(\mathrm{N}=264)$, and a group of people over 50 years who do not attend the University for Older People ( $\mathrm{N}=453)$. In a comparison of both groups, the results show that the students from the University for Older People obtain significantly lower global scores in daily memory, linguistic memory, sequential memory and memory of things.

Key words: University for older people, daily memory, active ageing, older people.

Correspondencia: José Jesús Gázquez Linares. Dpto de Psicología Evolutiva y de la Educación. Universidad de Almería. Ctra. de Sacramento, s/n. C.P: 04120 Almería (España). E-mail: jlinares@ual.es 
Una de las actuaciones más innovadoras desarrolladas en el ámbito de las personas mayores, iniciada en Francia en los años 60, y en la actualidad, con una implementación en todo el territorio español, son las Universidad de la Experiencia, aunque recibe diversas denominaciones, tales como: Universidad de Mayores, Programas de Mayores en la Universidad, Universidad de la Experiencia, Aulas para mayores, etc.; pero tienen en común, ser una actividad mediante la cual, la población mayor puede adquirir unos conocimientos universitarios ligados a diversas ramas del conocimiento y, en algunos casos (como en Almería y otras sedes de diversas ciudades), asistir a cualquier materia que se cursa en la Universidad. A pesar de ser una experiencia relativamente novedosa, posee un alto grado de satisfacción, no sólo entre los propios mayores que participan (Castellón, Gómez y Martos, 2004), sino también entre el propio profesorado y los coordinadores de los diversos programas.

La puesta en marcha de estas Aulas de Mayores, viene a desterrar algunos de los estereotipos más fuertemente acuñados respecto a la vejez, como son: su incapacidad para aprender algo nuevo o que la mayoría de los mayores son seniles (Fernández, 1992; Gázquez, Cangas, Lucas y Olivencia, 2005; Gázquez et al., 2009), y además se erigen como una alternativa para el envejecimiento activo de los mayores, siendo este uno de los retos de plena actualidad (OMS, 2002), que intenta poner freno al aumento de la dependencia dentro del conjunto de la población mayor (Puga, 2001), ya que, tal y como se ha demostrado en múltiples estudios, el grado de funcionalidad y la percepción de la propia salud aumentan a medida que lo hacen los años de educación formal (Blaxter, 1989; Ross y Mirowsky, 1999), disminuyendo por tanto, el grado de dependencia considerablemente (INE, 2008). Aunque esta relación entre el nivel educativo y la dependencia no es tan clara cuando hablamos de un nivel de dependencia considerado grave (Grundy y Glaser, 2000).

Los estudios más recientes, apoyan de manera consensuada, que la implicación en actividades de aprendizaje propicia una estimulación cognitiva continuada, además de proporcionar efectos protectores o estrategias compensatorias que ayudan a mitigar el declive cognitivo en la edad avanzada (Wight, Aneshensel y Seeman, 2002; Compton, Bachman, Brand y Avet, 2000). El impacto del programa universitario en las personas mayores, medido a partir del incremento en los niveles de integración y actividad social (Montoro y Pinazo, 2005), es particularmente relevante, dado que la actividad social ha sido a menudo vinculada con el bienestar psicológico de los mayores (Adelmann, 1994; Okun, Stock, Haring y Witter, 1984). La investigación al respecto, indica que, la capacidad de los aprendices mayores de beneficiarse de una mejora en la salud física, mental y social, se ve afectada por una continuada participación en actividades de aprendizaje (Dench y Regan, 2000; Purdie y BoultonLewis, 2003). 
Concretamente, respecto a los cambios en la memoria, asociados al envejecimiento y la efectividad de la intervención cognitiva, en la prevención del deterioro cognitivo, son múltiples los estudios realizados (Novoa, Juárez y Nebot, 2008). Pero, las intervenciones no cognitivas, también pueden tener efectos positivos sobre la cognición. Así por ejemplo, algunos estudios indican la existencia de una relación entre la presencia de recursos sociales y un descenso en la probabilidad de sufrir o presentar deterioro cognitivo (Glei et al., 2005; Verghese et al., 2003). En otros casos, se analiza la influencia de la integración social, entendida ésta, como la participación en actividades sociales, de ocio, interacción con familiares y amigos, etc., destacando que todo ello, dispensa apoyo emocional a los sujetos, lo que repercute en su función cognitiva (West y Yassuda, 2004). Por último, también hay estudios que relacionan la realización de actividad física con la mejora cognoscitiva de los mayores, aunque hay algunos, que no son concluyentes (Sturman et al., 2005), otros nos indican que la realización de ejercicio moderado, con cualquier frecuencia en las etapas adultas, está asociado con una menor probabilidad de sufrir deterioro cognitivo leve (Geda et al., 2010).

Prácticamente en estos estudios donde se analiza el beneficio de la intervención cognitiva, se realizan experimentos con programas de entrenamiento específicos, y son pocas las investigaciones centradas en otros aspectos, y menos aún en los Programas Universitarios para Mayores, donde no sólo se realiza una estimulación cognitiva, sino que también, la asistencia, lleva asociada otro tipo de aspectos no cognitivos (actividades sociales, de ocio, culturales, etc.). Así, el objetivo de este estudio es conocer si el alumnado de la Universidad de Mayores muestra mejores niveles en la memoria cotidiana que los mayores que no asisten a este programa de envejecimiento activo.

\section{METODO}

\section{Participantes}

La muestra está compuesta por un total de 717 sujetos mayores de 50 años de la provincia de Almería. Un análisis global, nos permite observar que la media de edad es de 65.5 años $(D T=8.83)$, de los cuales el 46,7\% (335 sujetos) son hombres con una media de edad de 65.3 años $(D T=8.97)$ y el $53.3 \%$ (382 sujetos) son mujeres cuya media de edad es de 65.7 años $(D T=8.70)$. En función de los grupos de edad quinquenales en los que ha sido agrupada la muestra observamos que el $9.9 \%$ (71 sujetos) tienen entre 50 y 54 años, el $22.6 \%$ (162 sujetos) entre 55 y 59 años, el $12.6 \%$ (90 sujetos) entre 60 y 64 años, el $19.5 \%$ (140 sujetos) entre 65 y 69 años, y los tres últimos grupos de 70 a 74 años, de 75 a 79 años y de 80 o más años, están compuestos respectivamente por el $19 \%$ (136 sujetos), el $10.9 \%$ (78 sujetos) y el $5.6 \%$ (40 sujetos). 
En función de que pertenezcan o no al Programa Universitario para Mayores, observamos en la siguiente tabla como el $42.4 \%$ de la muestra que asiste al Programa Universitario son hombres, y el 57.6\% Mujeres, con una media de edad de 68.8 años $(D T=6.39)$ y 66.8 años $(D T=6.30)$ respectivamente. El grupo que no pertenece al Programa Universitario se compone de 223 hombres (49.2\%) y de 230 mujeres $(50.8 \%)$, cuya media de edad es de 63.6 años $(D T=9.58)$ y 64.9 años $(D T=9.93)$ respectivamente. La media de edad de los 264 sujetos que pertenecen al Programa Universitario es de 67.7 años $(D T=6.40)$, distribuyéndose en función de su agrupación en grupos quinquenales del siguiente modo: el 3\% tienen entre 50 y54 años, el $7.6 \%$ entre 55 y 59 años, el $13.6 \%$ entre 60 y 64 años, el $37.9 \%$ entre 65 y 69 años, el $25 \%$ entre 70 y 74 años, el $9.8 \%$ entre 75 y 79 años, y finalmente, el $3 \%$ poseen más de 80 años. Por otro lado, la media de edad de los 453 sujetos que componen el grupo que no pertenece al Programa Universitario es de 64.3 años $(D T=9.77)$, y se distribuyen entre los grupos del siguiente modo: el $13.9 \%$ tiene entre 50 y 54 años, el $31.3 \%$ entre 55 y 59 años, el $11.9 \%$ entre 60 y 64 años, el $8.8 \%$ entre 65 y 69 años, el $15.5 \%$ entre 70 y 74 años, el $11.5 \%$ entre 75 y 79 años, y finalmente, tiene más de 80 años el $7.1 \%$ de la muestra.

Tabla 1. Descriptivos de los dos grupos en función del género y los grupos de edad

\begin{tabular}{|c|c|c|c|c|c|c|c|c|c|c|}
\hline \multirow{2}{*}{$\begin{array}{c}\text { Programa } \\
\text { Universitario }\end{array}$} & & \multicolumn{2}{|c|}{ Género } & \multicolumn{7}{|c|}{ Edad } \\
\hline & & Masculino & Femenino & 50-54 años & 55-59 años & 60-64 años & 65-69 años & 70-74 años & 75-79 años & 80 o más \\
\hline \multirow{2}{*}{ SI } & $\mathrm{N}$ & 112 & 152 & 8 & 20 & 36 & 100 & 66 & 26 & 8 \\
\hline & $\%$ & $42.4 \%$ & $57.6 \%$ & $3 \%$ & $7.6 \%$ & $13.6 \%$ & $37.9 \%$ & $25 \%$ & $9.8 \%$ & $3 \%$ \\
\hline \multirow{2}{*}{ NO } & $\mathrm{N}$ & 223 & 230 & 63 & 142 & 54 & 40 & 70 & 52 & 32 \\
\hline & $\%$ & $49.2 \%$ & $50.8 \%$ & $13.9 \%$ & $31.3 \%$ & $11.9 \%$ & $8.8 \%$ & $15.5 \%$ & $11.5 \%$ & $7.1 \%$ \\
\hline
\end{tabular}

Finalmente, el análisis de los dos grupos en función del nivel de estudios realizado en la tabla 2, nos muestra como en el grupo que asiste al Programa Universitario el mayor número de sujetos, el 25\%, posee Bachiller, seguido del $18.9 \%$ que son Diplomados, el $17.4 \%$ que poseen estudios Primarios, el $12.9 \%$ que son Licenciados, y finalmente, el $1.5 \%$ que tienen Formación Profesional, y ese mismo porcentaje no posee estudios. Dentro del grupo que no asiste al Programa Universitario la muestra se distribuye en función de su nivel de estudios del siguiente modo: el 34\% posee estudios Primarios, el 22.5\% no posee estudios, el $14.6 \%$ son Diplomados, el $14.3 \%$ poseen Bachiller, el $4.4 \%$ Formación Profesional, y finalmente, sólo el $2.9 \%$ de este grupo son Licenciados.

Tabla 2. Descriptivos de los dos grupos en función del nivel de estudios

\begin{tabular}{cccccccc}
\hline \multirow{2}{*}{$\begin{array}{c}\text { Programa } \\
\text { Universitario }\end{array}$} & & \multicolumn{6}{c}{ Nivel de estudios } \\
\cline { 2 - 8 } & Sin estudios & Primarios & Bachiller & Diplomado & Licenciado & Formación Profesional \\
\hline \multirow{2}{*}{ SI } & $\mathrm{N}$ & 4 & 46 & 66 & 50 & 34 & 4 \\
& $\%$ & $1.5 \%$ & $17.4 \%$ & $25 \%$ & $18.9 \%$ & $12.9 \%$ & $1.5 \%$ \\
\hline \multirow{2}{*}{ NO } & $\mathrm{N}$ & 102 & 154 & 65 & 66 & 13 & 20 \\
& $\%$ & $22.5 \%$ & $34 \%$ & $14.3 \%$ & $14.6 \%$ & $2.9 \%$ & $4.4 \%$ \\
\hline
\end{tabular}




\section{Instrumentos}

Cuestionario de Memoria Cotidiana (CMC) (Cornish, 2000; traducido y adaptado al castellano por D. Manga: Universidad de León): permite, tal y como su propio nombre indica, evaluar la memoria cotidiana, para lo cual consta de varias subescalas en las que se evalúa la memoria espacial, la memoria lingüística, la memoria secuencial, la memoria de cosas, la memoria Cotidiana Compuesto y la memoria Global. Establece cinco alternativas de respuesta ( $1=$ Nunca o casi nunca; $2=$ Pocas veces; $3=$ Algunas veces; $4=$ Con frecuencia $;$ y $5=$ Muchas veces $)$ donde se pide valorar la frecuencia con que se dan las diferentes situaciones todas ellas respecto a situaciones negativas o de olvido, por lo que las bajas puntuaciones son positivas respecto al nivel mostrado en los diferentes tipos de memoria evaluados.

Test del Dibujo del Reloj (TDR) (Thalman et al., 1996): es una prueba de detección (screening) sencilla, rápida y de fácil aplicación para valorar el estado cognitivo del sujeto que se utiliza muy a menudo para el estudio y valoración de las enfermedades neurodegenerativas, especialmente para la demencia y la enfermedad de Alzheimer. De forma concreta, evalúa diferentes mecanismos implicados en la ejecución de las tareas, fundamentalmente: funciones visoperceptivas, visomotoras $\mathrm{y}$ visoconstructivas, planificación y ejecución motoras.

\section{Procedimiento}

Por un lado, se seleccionó al azar una muestra de 600 personas mayores de 50 años que no pertenecían ni habían pertenecido en ningún momento de su vida al Programa Universitario de Mayores, de las que aceptaron participar en la investigación 453 personas.

Por otro lado, se solicitó permiso al responsable del Programa Universitario de Mayores: Ciencia y Experiencia de la Universidad de Almería, para la aplicación de los cuestionarios seleccionados a los alumnos que llevaban asistiendo al programa más de cinco años, es decir, habían completado su formación durante los dos primeros ciclos o estaban finalizando segundo ciclo, al final del quinto año. La muestra de este grupo fue seleccionada entre las sedes de Almería, Roquetas de Mar y Vera, contando en esos cursos con un total de 397 personas que se encontraban en esos niveles educativos, de los cuales cumplimentaron los cuestionarios un total de 264 sujetos.

Para la obtención de los datos se ha instruyó a cuatro personas que fueron las encargadas de aplicar el cuestionario a la muestra que participó en el estudio. Una vez se pasaron las correspondientes pruebas y fueron corregidas, se procedió a la elaboración e introducción de los datos en la respectiva base de datos, y se procedió a su análisis a través del programa estadístico SPSS 15.0. Antes de introducir los datos debemos indicar que el Test del Dibujo del Reloj (TDR) fue aplicado a toda la muestra con el objeto de detectar de forma sencilla y rápida un posible deterioro cognitivo, con lo que aquellos 
sujetos, que este test nos mostraban indicios de deterioro, fueron eliminados de la muestra.

\section{RESULTADOS}

En todos los casos en los que analizamos la diferencia de puntuaciones medias, con el objetivo de conocer si existen diferencias entre las puntuaciones del grupo de sujetos que pertenecen a la Universidad de Mayores y del grupo que no pertenece a este programa, será utilizada la prueba $t$ para el análisis de la diferencia entre las medias.

Así en la tabla 3, observamos que los mayores asistentes a la Universidad de Mayores obtienen puntuaciones significativamente menores en la puntuación global del CMC $(t(1.699)=-3.017, p=0.003)$, en la memoria lingüística $(t(1.699)=-3.060, p=$ $0.002)$, secuencial $(t(1.699)=-3.720, p=0.000) \mathrm{y}$ de $\operatorname{cosas}(t(1.699)=-2.105, p=0.036)$, siendo destacable además, que en la memoria espacial, donde no se aprecian diferencias significativas $(t(1.699)=-0.265, p=0.791)$, las puntuaciones medias de estos también son inferiores a las de los mayores que no pertenecen al Programa.

Tabla 3. Descriptivos y diferencia de medias en función de la pertenencia o no al Programa Universitario

\begin{tabular}{|c|c|c|c|c|c|c|}
\hline & \multicolumn{6}{|c|}{ Programa Universitario } \\
\hline & \multicolumn{3}{|c|}{$\mathrm{Si}$} & \multicolumn{3}{|c|}{ No } \\
\hline & $N$ & $M$ & $\overline{D T}$ & $N$ & $M$ & $\overline{D T}$ \\
\hline Memoria Espacial & 248 & 9.90 & 3.46 & 453 & 9.98 & 3.46 \\
\hline Memoria Lingüística & 248 & 11.21 & 3.57 & 453 & 12.20 & 4.37 \\
\hline Memoria Secuencial & 248 & 8.71 & 2.92 & 453 & 9.76 & 3.88 \\
\hline Memoria de cosas & 248 & 6.97 & 2.36 & 453 & 7.46 & 3.27 \\
\hline Memoria Global & 248 & 51.02 & 14.77 & 453 & 55.15 & 18.52 \\
\hline
\end{tabular}

Un análisis en función del género (Tabla 4 y 5) nos muestra en los hombres diferencias significativas entre los que asisten al Programa y los mayores que no lo hacen en la puntuación global del CMC $(t(1.329)=-3.317, p=0.001)$, así como para la memoria lingüística $(t(1.329)=-3.253, p=0.001)$, secuencial $(t(1.329)=-3.017, p=$ $0.002)$ y de cosas $(t(1.329)=-2.002, p=0.046)$, siendo significativamente superiores, las puntuaciones de los mayores que no asisten al programa. Nuevamente, en la memoria espacial, son los mayores universitarios los que presentan puntuaciones inferiores, aunque no sea significativa esa diferencia con respecto a los no universitarios $(t(1.329)=$ $-0.824, p=0.410)$.

Respecto al género femenino, sólo se encuentran diferencias significativas en las puntuaciones medias entre el grupo de alumnas de la Universidad y el grupo de mayores no perteneciente al programa, respecto a la memoria secuencial $(t(1.368)=$ 2.418, $p=0.016$ ), siendo superior la puntuación de este último grupo. Aunque sin diferencias significativas, las puntuaciones de las alumnas del programa universitario, 
son inferiores en la memoria lingüística $(t(1.368)=-1.666, p=0.097)$, de cosas $(t(1.368)=$ $-1.234, p=0.218)$ y global $(t(1.368)=-1.389, p=0.166)$, salvo en la memoria espacial $(t(1,368)=0,319, p=0,750)$, donde es mínimamente superior la puntuación media del grupo de mujeres que no asisten a la Universidad de Mayores.

Tabla 4. Género masculino. Descriptivos y diferencia de medias en función de que pertenezcan o no al Programa Universitario

\begin{tabular}{|c|c|c|c|c|c|c|}
\hline \multirow{3}{*}{ Aspectos Evaluados } & \multicolumn{6}{|c|}{ Género Masculino } \\
\hline & \multicolumn{3}{|c|}{ Programa Universitario } & \multicolumn{3}{|c|}{ No Programa Universitario } \\
\hline & $N$ & $M$ & $D T$ & $N$ & $M$ & $D T$ \\
\hline Memoria Espacial & 108 & 9.6 & 2.57 & 223 & 9.9 & 3.76 \\
\hline Memoria Lingüística & 108 & 10.2 & 2.61 & 223 & 11.6 & 4.18 \\
\hline Memoria Secuencial & 108 & 8.1 & 2.25 & 223 & 9.4 & 4.21 \\
\hline Memoria de cosas & 108 & 6.4 & 2.23 & 223 & 7.2 & 3.38 \\
\hline Memoria Global & 108 & 47.2 & 10.73 & 223 & 53.6 & 18.68 \\
\hline
\end{tabular}

Tabla 5. Género femenino. Descriptivos y diferencia de medias en función de que pertenezcan o no al Programa Universitario

\begin{tabular}{lcccccc}
\hline \multirow{2}{*}{ Aspectos Evaluados } & \multicolumn{6}{c}{ Género Femenino } \\
\cline { 2 - 7 } & $N$ & $M$ & $D T$ & $N$ & $M$ & $D T$ \\
\cline { 2 - 7 } & 140 & 10.1 & 4.01 & 230 & 10.0 & 3.16 \\
\hline Memoria Espacial & 140 & 11.9 & 4.01 & 230 & 12.7 & 4.49 \\
Memoria Lingüística & 140 & 9.2 & 3.27 & 230 & 10.1 & 3.52 \\
Memoria Secuencial & 140 & 7.3 & 2.39 & 230 & 7.7 & 3.15 \\
Memoria de cosas & 140 & 53.9 & 16.72 & 230 & 56.6 & 18.31 \\
Memoria Global & & &
\end{tabular}

El análisis de los resultados del Cuestionario de Memoria Cotidiana en función de la edad, nos muestra con la realización de la prueba de correlación de Pearson (Tabla 6), que, salvo en el grupo de alumnos y respecto a la memoria secuencial, en todos los casos se establece una correlación positiva entre el aumento de la edad y el aumento de las puntuaciones en memoria espacial, linguiística, de cosas y global, así como para la secuencial respecto a los mayores que no pertenecen al Programa Universitario para mayores.

Con el objetivo de conocer si los niveles de la variable dependiente, en este caso, la puntuación obtenida en el Cuestionario de Memoria Cotidiana (Global), se ve influenciada por la pertenencia o no al Programa Universitario y por el nivel de estudios de los mayores, así como por la interacción entre ambas variables, se realiza una ANOVA de dos factores. Inicialmente, tal y como vemos en la gráfica 1, podemos indicar que estas diferencias se dan en las personas sin estudios, con estudios primarios, de formación profesional y los diplomados.

Un análisis de la interacción significativa entre ambos grupos (Nivel de Estudios y Programa Universitario), tal y como muestra la tabla 7, a través de la prueba 
$T$ de Student para muestras independientes en función de que pertenezcan o no al Programa Universitario, nos muestra que estadísticamente las diferencias son significativas entre ambos grupos,.en las personas sin estudios $(t(1,194)=-6,997, p=$ $0.000)$, con estudios primarios $(t(1.194)=-2.337, p=0.021)$, con Formación Profesional $(t(1.22)=-3.652, p=0.002), y$ los Diplomados $(t(1.110)=3.104, p=0.003)$, en los tres primeros niveles educativos son los mayores que no pertenecen a la Universidad de Mayores los que poseen significativamente mayores puntuaciones que los mayores universitarios; mientras que es al contrario entre los Diplomados, donde son los que sí pertenecen a la Universidad de Mayores los que puntúan significativamente por encima respecto a los mayores no universitarios. En los niveles educativos de Bachillerato $(t(1.125)=-0.156, p=0.877)$ y Licenciatura $(t(1.45)=-0.858, p=0.403)$ no se encuentran diferencias significativas entre las puntuaciones de Memoria Cotidiana Global del grupo de mayores universitario y no universitario.

Tabla 6. Correlación de Pearson en función de la edad según pertenezcan o no al Programa Universitario

\begin{tabular}{lcccc}
\hline \multirow{2}{*}{ Aspectos Evaluados } & \multicolumn{2}{c}{ Programa Universitario } & \multicolumn{2}{c}{ No Programa Universitario } \\
\cline { 2 - 5 } & $\begin{array}{c}\text { Correlación } \\
\text { de Pearson }\end{array}$ & $\begin{array}{c}\text { Sig. } \\
\text { (bilateral) }\end{array}$ & $\begin{array}{c}\text { Correlación de } \\
\text { Pearson }\end{array}$ & $\begin{array}{c}\text { Sig. } \\
\text { (bilateral) }\end{array}$ \\
\hline Memoria Espacial & $.195^{* *}$ & .002 & $.172^{* *}$ & .000 \\
Memoria Lingüística & $.197^{* *}$ & .002 & $.161^{* *}$ & .001 \\
Memoria Secuencial & .111 & .081 & $.132^{* *}$ & .005 \\
Memoria de cosas & $.213^{* *}$ & .001 & $.233^{* *}$ & .000 \\
Memoria Global & $.174^{* *}$ & .006 & $.176^{* *}$ & .000 \\
\hline ** La correlación es significativa al nivel 0.01 (bilateral). & & & \\
$*$ La correlación es significativa al nivel 0.05 (bilateral). & & &
\end{tabular}

Gráfica 1. Memoria Cotidiana Global. Programa Universitario - Nivel de Estudios

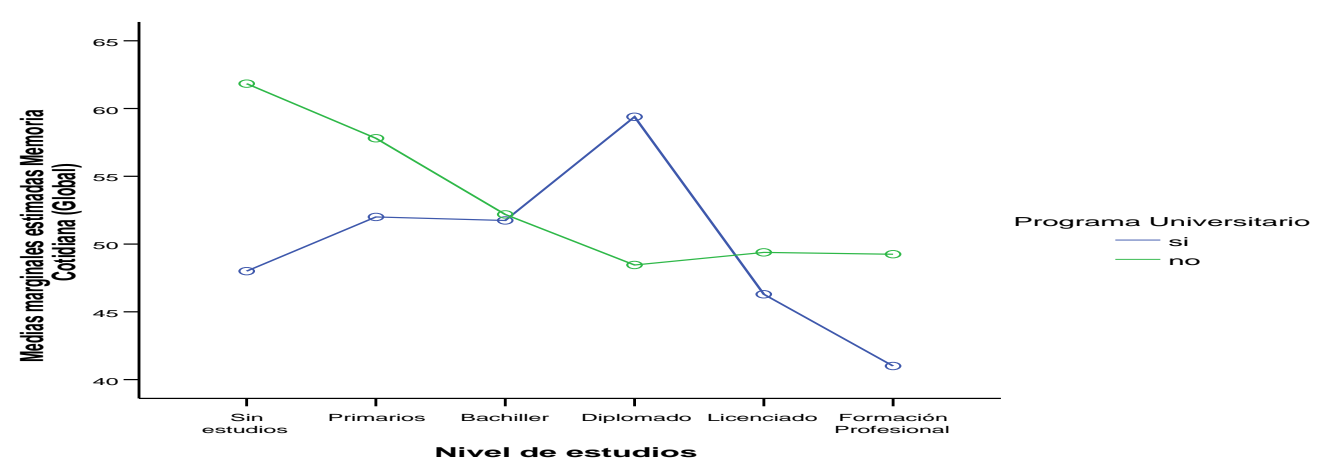


Tabla 7. Memoria Cotidiana Global. ANOVA dos colas. Programa Universitario, Nivel de Estudios e

\begin{tabular}{lccccc}
\multicolumn{7}{c}{ Interacción (PU x NE) } \\
\hline Suma de cuadrados & $g l$ & Media cuadrática & $F$ & Sig. \\
\hline Programa Universitario & 548.650 & 1 & 548.650 & 1.807 & .179 \\
\hline Nivel de Estudios & 2586.099 & 5 & 517.220 & 1.703 & .132 \\
\hline (PU x NE) & 5410.626 & 5 & 1082.125 & 3.564 & .003 \\
\hline (PU=Programa Universitario; NE=Nivel de Estudios) & & &
\end{tabular}

\section{DISCUSION}

Tal y como hemos indicado, las Universidades de Mayores son una de las muchas iniciativas de envejecimiento activo, que tienen como objetivo la optimización del proceso de envejecimiento. Así observamos a través del presente estudio las repercusiones que la asistencia a este programa posee sobre la memoria de los mayores, obteniendo puntuaciones significativamente menores, y por tanto, mejores niveles, en la puntuación global del Cuestionario de Memoria Cotidiana (CMC), así como en la memoria lingüística y secuencial,.siendo destacable además, que en la memoria espacial y de cosas, no se aprecian diferencias significativas, pero las puntuaciones medias de estos, también son inferiores a las de los mayores que no pertenecen al programa.

Un análisis en función del nivel de estudios, que en muchos casos también ha estado ligado al estado cognitivo, indicando que existe una relación lineal entre el nivel de estudios y el deterioro cognitivo (Blaxter, 1989; Ross y Mirowsky, 1999), los resultados nos muestran que sólo entre los Diplomados son los que sí pertenecen a la Universidad de Mayores los que puntúan significativamente por encima respecto a los mayores no universitarios, por tanto, presentan peores niveles cognitivos. En el caso de los que no tienen estudios, los que tienen estudios primarios y Formación Profesional, son los mayores que pertenecen a la Universidad de Mayores los que significativamente mejores niveles cognitivos muestran.

Tal y como era de esperar, en función de la edad, salvo en el grupo de alumnos de la Universidad de Mayores respecto a la memoria secuencial, en todos los casos se establece una correlación positiva entre el aumento de la edad y el aumento de las puntuaciones en memoria espacial, lingüística, de cosas y global, pudiendo indicar que la Universidad de Mayores mitiga los efectos del envejecimiento respecto a la memoria secuencial, al igual que los múltiples programas de actuación y estimulación que se están llevando a cabo (Wight, Aneshensel y Seeman, 2002; Compton, Bachman, Brand y Avet, 2000).

Es como si a los ancianos les importara menos su rendimiento y se esforzaran menos en retener, e incluso a veces parece que se les ha olvidado cómo retenerlo, pero cuando el material les interesa, significa algo para ellos, se les dan pistas o se les refresca cómo organizarlo, vuelven a mostrar una memoria similar a la que anteriormente 
mostraban (Calero, 2000), quizás este sea uno de los efectos de la asistencia a la Universidad de Mayores, el interés y la motivación para los aprendizajes, que finalmente suponen un ejercicio continuado de entrenamiento cognitivo.

Por último, indicar que en este sentido, sería necesario tener en cuenta que el diseño de la investigación se ha realizado de modo transversal mediante la comparación de dos grupos, uno perteneciente a la Universidad de Mayores y otro de adultos externos, con lo que quizás existan entre ambos grupos diferencias previas, no de carácter cognitivo, sino psicológicas y sociales, que estén influyendo la presencia de esos mejores niveles cognitivos en los alumnos de la Universidad de Mayores, y no se deba simplemente a la participación en el programa durante los últimos cinco años. Para ello, una futura línea de investigación pasaría por la realización de un estudio longitudinal y un análisis de las características no cognitivas iniciales.

\section{Agradecimientos}

A la Consejería de Bienestar e Igualdad Social de la Junta de Andalucía por la financiación del proyecto. Al Vicerrectorado de Cultura, Extensión Universitaria y Deportes de la Universidad de Almería, así como al alumnado de la Universidad de Mayores, por el interés y su participación en dicho trabajo.

\section{REFERENCIAS}

Adelmann, P.K. (1994). Multiple roles and psychological well-being. Journal of Gerontology, 49(6), S277-S285.

Blaxter, M. (1989). A comparison of measures of inequality in morbidity. En J. Fox (Ed.), Health Inequalities in European Countries (pp. 199-230). Gower: Aldershot.

Calero, M.D. (2000). Psicología de la vejez: el funcionamiento cognitivo. En R. FernándezBallesteros (dir.), Gerontología Social. Madrid: Pirámide.

Castellón, A., Gómez, M.A. y Martos, A. (2004). Análisis de la satisfacción en los mayores de la Universidad de Granada. Revista Multidisciplinar de Gerontología, 14(5), 252-257.

Compton, D.M., Bachman, L.D., Brand, D. \& Avet, T.L. (2000). Age-associated changes in cognitive function in highly educated adults: Emerging myths and realities. International Journal of Geriatric Psychiatry, 15, 75-85.

Cornish, I.M. (2000). Factor structure of the Everyday Memory Questionnaire. British Journal of Psychology, 91, 427-438.

Dench, S. \& Regan, J. (2000). Learning in later life: Motivation and impact. Nottingham: DfEE.

Fernández, R. (1992). Mitos y realidades sobre la vejez y la salud. Madrid: Fundación Caja de Madrid.

Gázquez, J.J., Cangas, A.J., Lucas, F. y Olivencia, J.J. (2005). Estereotipos sobre la vejez en estudiantes universitarios. En I. Ruiz, F.V. Castro, A. Ventura, J.A. Barrio y M.I. Fajardo (Coords.), Necesidades Educativas Específicas: ¿hay respuestas? (pp. 285291). Psicoex: Badajoz.

Gázquez, J.J., Pérez, M.C., Fernández, M., González, L., Ruiz, I. \& Díaz, A. (2009). Old-age stereotypes related to the gerontology education: an intergenerational study. European Journal of Education and Psychology, 2(3), 263-273. 
Geda, Y.E., Roberts, R.O., Knopman, D.S., Christianson, T.J.H., Pankratz, V.S., Ivnik, R.J., Boeve, B.F., Tangalos, E.G., Petersen, R.C. \& Rocca, W.A. (2010). Physical Exercise, Aging, and Mild Cognitive Impairment. A Population-Based Study. Archives of Neurology, 67(1), 80-86.

Glei, D.A., Landau, D.A., Goldman, N., Chuang, Y., Rodríguez, G. \& Weinstein, M. (2005). Participating in social activities helps preserve cognitive function: an analysis of a longitudinal, population-based study of the elderly. International Journal of Epidemiology, 34(4), 864-871.

Grundy, E. \& Glaser, K. (2000). Socio-demographic differences in the onset and progression of disability in early old age: a longitudinal study. Age and Ageing, 29, 149-157.

INE (2008). Encuesta sobre Discapacidades, Deficiencias y Estado de Salud (EDDS). Madrid: INE.

Montoro, J. \& Pinazo, S. (2005)..Evaluating Social Integration and Psychological Outcomes for Older Adults Enrolled at a University Intergenerational Program. Journal of Intergenerational Relationships, 3(3), 65-81.

Novoa, A.M., Juárez, O. y Nebot, M. (2008). Efectividad de las intervenciones cognitivas en la prevención del deterioro de la memoria en las personas mayores sanas. Gaceta Sanitaria, 22(5), 474-482.

Okun, M., Stock, W.A., Haring, M.J. \& Witter, R.A. (1984). The social activity/subjective wellbeing relation. Research on Aging, 6(1), 45-65.

OMS (2002). Envejecimiento activo: un marco político. Revista Española de Geriatría y Gerontología, 37(S2), 74-105.

Puga, M.D. (2001). Dependencia y Necesidades Asistenciales de los Mayores en España. Previsión al año 2010. Madrid: Fundación Pfizer.

Purdie, N. \& Boulton-Lewis, G. (2003). The learning needs of older adults. Educational Gerontology, 29, 129-149.

Ross, C.E. \& Mirowsky, J. (1999). Refining the association between education and health: the effects of quantity, credential, and selectivity. Demography, 36(4), 445-460.

Sturman, M.T., Morris, M.C., Mendes de León, C.F., Bienias, J.L., Wilson, R.S. \& Evans, D.A. (2005). Physical Activity, Cognitive Activity, and Cognitive Decline in a Biracial Community Population. Archives of Neurology, 62(11), 1750-1754.

Verghese, J., Lipton, R.B., Katz, M.J., Hall, C.B., Derby, C.A., Kuslansky, G., Ambrose, A.F., Sliwinski, M. \& Buschke, H. (2003). Leisure activities and the risk of dementia in the elderly. The New England Journal of Medicine, 348, 2508-2516.

Wight, R.G., Aneshensel, C.S. \& Seeman, T.E. (2002). Educational attainment, continued learning experience, and cognitive function among older men. Journal of Aging and Health, 14(2), 211-236.

Recibido: 11 de julio de 2009 Recepción Modificaciones: 12 de diciembre de 2009

Aceptado: 15 de enero de 2010 